\title{
A Multiphysics Model of Myoma Growth
}

\author{
Dominik Szczerba $^{1}$, Bryn A. Lloyd ${ }^{1}$, Michael Bajka² ${ }^{2}$, and Gábor Székely ${ }^{1}$ \\ ${ }^{1}$ Computer Vision Laboratory, ETH, CH-8092 Zürich, Switzerland \\ dominik.szczerba@vision.ee.ethz.ch \\ ${ }^{2}$ Clinic of Gynecology, University Hospital of Zürich, Switzerland
}

\begin{abstract}
We present a first attempt to create an in-silico model of a uterine leiomyoma, a typical exponent of a common benign tumor. We employ a finite element model to investigate the interaction between a chemically driven growth of the pathology and the mechanical response of the surrounding healthy tissue. The model includes neoplastic tissue growth, oxygen and growth factor transport as well as angiogenic sprouting. Neovascularisation is addressed implicitly by modeling proliferation of endothelial cells and their migration up the gradient of the angiogenic growth factor, produced in hypoxic regions of the tumor. The response of the surrounding healthy tissue in our model is that of a viscoelastic material, whereby a stress exerted by expanding neoplasm is slowly dissipated. By incorporating the interplay of four underlying processes we are able to explain experimental findings on the pathology's phenotype. The model has a potential to become a computer simulation tool to study various growing conditions and treatment strategies and to predict post-treatment conditions of a benign tumor.
\end{abstract}

\section{Introduction}

Tumor growth is one of the leading diseases in humans. Whereas aggressively growing malignant tumors lead to anarchic morphological patterns also breaking into the surroundings, there are usually regularly developed structures respecting borders with a well developed network of vessels in slowly growing benign tumors. These structure-bearing characteristics predestine benign tumors to be analyzed by creating in silico models of the growth process. Better understanding of tumor development might help to optimize the existing therapeutic procedures, design new ones, or even provide post-treatment predictions. Uterine leiomyomas (fibroids, a typical exponent of a benign tumor) are the most common uterine neoplasm affecting more than $30 \%$ of women older than 30 years of age. Especially submucous leiomyoma are of primary clinical interest because they often distort the uterine cavity causing serious gynecological disturbances including pelvic pain, bleeding disorders and sterility. Fibroids are the dominating benign pathology of the uterus. They are predominantly composed of smooth muscle and a variable amount of fibrous tissue. Despite the amount of research in this area, the exact etiology and pathogenesis of a myoma is not known. It is assumed that the genesis is initiated by regular muscle cells with increased growth potential and that the growth of myomas is driven by estrogen and local 
growth factors. In general, a myoma grows slowly but continuously until the beginning of menopause. The increase of volume by a factor of two usually takes several months or years. A myoma has a much stronger tendency to keep its shape than any of the tissues surrounding it, as it is composed of very dense fibrotic tissue. There is no real capsule around a myoma which is only surrounded by a clustered myometrium. Most often myomas are classified depending on their position relative to the uterine wall: intramural, subserosal (visible from the abdominal cavity) or submucosal (visible from the uterine cavity). Latter are discerned in pedunculated myomas (type 0), predominantly intracavitary myomas (type I, forming an acute angle with the uterine wall, intracavitary portion $>50 \%$ ) and predominantly intramural myomas (type II, forming an obtuse angle with the uterine wall, intracavitary portion $<=50 \%$ ). These different degrees of intracavitary protrusion may result from myoma migration trough the healthy surrounding muscular mesh during the growth process whereas the exact mechanism remains unclear. The endometrium is a reactive tissue carrying viscoelastic characteristics covering the entire uterine cavity including protruding myomas of any degree. It may be stretched and thinned out until its blood supply may be affected and punctual necrosis of the endometrium may occur. Blood supply to the fibroids is provided by one or two large vessels ([1]), whereas [2 found that small fibroids $(1-3 \mathrm{~mm})$ were avascular, in larger ones $(<1 \mathrm{~cm})$ a few small vessels invaded the lesion from the periphery, and the largest fibroids $(>1 \mathrm{~cm})$ contained irregular networks of blood vessels with density similar to or lower than in normal myometrium, being surrounded by an extremely dense vascular layer. Walocha concluded that, during development of leiomyoma, the pre-existing blood vessels undergo regression and new vessels invade the tumor from the periphery, where intense angiogenesis, probably promoted by growth factors secreted by the tumor, leads to the formation of a vascular capsule, responsible for supply of blood to the growing tumor. We aim at a physically correct simulation, while relying on realistic physiological parameters when describing the underlying processes. Only such a low-level modeling paradigm will have sufficient predictive power required to study e.g. effects of drug administration or the influence of various interventional strategies on the surgical outcome. In this paper we present a first attempt to create a coupled mechano-chemical model of a growing leiomyoma. Followed by a literature overview of the morphological and physiological tumor development and previously proposed methods for simulation of tumor growth, we present our multiphysics model. Results generated by its implementation demonstrate the feasibility of our approach.

\section{Literature Overview}

Tumor development has been extensively studied for the last 3 decades. An overview of the available literature relevant to our approach is given in previous works $(3], 4], 5])$. We only briefly comment on the presented approaches, that we arbitrarily group into the following categories: 1) mathematical models, 2) cellular automata, 3) finite element methods. In general, mathematical models 
are very interesting to study cellular metabolism and the temporal and spatial dynamics of tumor development, they are, however, often limited in scope (e.g. do not cover tissue mechanics) while being already significantly complex in the mathematical formulation used. Moreover, their mathematical sophistication is not always readily implementable for real-life clinical problems defined by patient-specific finite element discretizations of e.g. volumetric radiological data. Cellular automaton approaches are demonstrated to be particularly well suited to model dynamics of developing pathologies while being relatively accessible in formulation. Such models, however, typically do not address the important mechanical interactions between the tumor and healthy tissue. We identify our approach largely with the third group, adopting the modeling techniques from solid and fluid mechanics as well as chemical engineering. This is particularly true for the strain-induced cellular response, gaining acceptance as an influential player in tissue development as shown in 6. With finite element models we can account for mechanical tissue deformations induced by developing pathologies and capture its interplay with the chemical environment. In this context multiphysics models, i.e. models combining a few independent physical phenomena into one computationally coherent simulation, become of particular interest. Feasibility of such an approach has recently been demonstrated by 4 on the example of micro-vessel growth and remodeling. We propose to apply the same modeling paradigm to simulate the development of a leiomyoma.

\section{The Multiphysics Model}

We initialize the pathology growth inside a hosting tissue (myometrium) and realize its development as a response to activating factors secreted by the growing tumor. The dividing cells acquire their oxygen and nutrients by intra-cellular diffusion-dominated supply (corresponding to small fibroids 1-3 mm ([2])). Such development will stop when a certain critical mass has been reached, as diffusionbased transport is no longer efficient for proliferation-dominated growth. In such cases the tumor will attract external blood supply from the adjacent parent vessels to acquire necessary oxygen and nutrients (corresponding to larger fibroids ([2])). The formation of blood vessels during angiogenesis in general - healthy or cancerous - is a process where capillary sprouts depart from pre-existing parent vessels in response to externally supplied chemical stimuli. By means of endothelial cell proliferation and migration the sprouts then organize themselves into a branched, connected network structure, created in order to feed the developing tumor. A detailed description of this process in context of computer modeling is given by 3 . The novelty of our model compared to the one described therein is not only to use the delivered nutrients to explicitly control the growth, but couple the growth back to the vessel network behavior via secretion of tumor angiogenesis growth factors (AGF). The components of this linked bio-chemomechanical model are described in the following sections. 


\subsection{Tumor Neovascularization}

We start our modeling from the widely accepted assumption that the initial response of the endothelial cells to the angiogenic growth factors is due to chemotaxis, enforcing cell migration towards the tumor or ischemic cell. Once secreted, the growth factors diffuse from the tumor (domain $\Omega_{2}$ ) into the surrounding tissue (domain $\Omega_{1}$ ) establishing a certain concentration gradient between the chemical source and the parent vessels. As the endothelial cells migrate through the extracellular matrix in response to this gradient there is some uptake and binding of the growth factors by the cells ([7). Therefore, this process can be modeled by a diffusion equation with a natural decay term:

$$
\begin{aligned}
\frac{\partial c_{1}}{\partial t} & =D_{1} \nabla^{2} c_{1}-R_{1} c_{1} \\
c_{1} & =\alpha_{1} H^{-} \mid \partial \Omega_{12} \\
H^{-} & =\int_{c_{3} \leq c_{3}^{t h}} d \Omega_{2}
\end{aligned}
$$

with $c_{1}$ being the chemical concentration of the growth agent, $D_{1}$ its diffusion constant and $R_{1}$ decay rate. The amount of the growth factor released depends (here, linearly) on the amount of tumor cells suffering from hypoxia $\mathrm{H}^{-}$, with a minimum required oxygenation threshold $c_{3}^{t h}$. Once the distribution of the growth agent has been initiated, endothelial cells start to respond to the stimulus. The endothelial density evolution is governed by a conventional convectiondiffusion equation that we write as:

$$
\begin{aligned}
\frac{\partial c_{2}}{\partial t} & =D_{2} \nabla^{2} c_{2}+\nabla \cdot\left(c_{2} \boldsymbol{u}\right) \\
\boldsymbol{u} & =\left(\frac{k_{0} k_{1}}{k_{1}+c_{1}} \nabla c_{1}\right)
\end{aligned}
$$

with $c_{2}$ being the endothelial cell density and $D_{2}, R_{2}$ positive constants. Note the dependence of the convective velocity $\boldsymbol{u}$ on the growth factor concentration $c_{1}$. Now that a vascular system has changed according to the growth agent, a new distribution of oxygen results, governed by:

$$
\begin{aligned}
\frac{\partial c_{3}}{\partial t} & =D_{3} \nabla^{2} c_{3}-R_{3} c_{3} \\
c_{3} & =\alpha_{3} c_{2} \mid \partial \Omega_{12}
\end{aligned}
$$

with $D_{3}$ being the diffusion constant and $R_{3}$ the reaction rate. Here, the amount of oxygen delivered to the tumor depends linearly on the density of endothelial cells, assumed to form predominantly capillaries close to the tumor.

\subsection{Tissue Mechanics}

We base our modeling on experimental observations of mechanic tissue responses to chemical growth factors, see e.g. [6. Production of such growth factors in our 
model is a result of ischemia, as described in the preceding section. As the growth itself results largely from increased proliferation rate, we can model it as initial strain condition:

$$
\epsilon_{0}(t)=\frac{\int_{t}^{t+\Delta t} \frac{\partial N}{\partial t} d t}{N} \cong \frac{N(t+\Delta t, x, \theta)-N(t, x, \theta)}{N(t, x, \theta)}
$$

where $N(t, x, \theta)$ is the number of cells in a finite element at time $t$ and position $x$. For a constant growth rate the strain is $\frac{\Delta t}{T_{2}}$, where $T_{2}$ is the cell population doubling time. The proliferation rate and, therefore, the number of cells depends on certain environmental factors $\theta$ such as oxygen availability. Based on descriptions in the literature $([8])$ we have defined this dependency with a piece-wise linear function $\left(\epsilon_{0}=\frac{\Delta t}{T_{2}} f\left(c_{3}(t)\right)\right)$, which relates the deviation from normal oxygen concentration to the amount of growth. A step function around a hypoxia threshold $c_{3}$ has been assumed, thus

$$
\begin{aligned}
\epsilon_{0} & =\alpha_{4} H^{+} \\
H^{+} & =\int_{c_{3}>c_{3}^{t h}} d \Omega_{2}
\end{aligned}
$$

which aims to approximate the fact that cells receiving enough oxygen will proliferate resulting in stress both in the tumor and the surrounding tissue. To quantify this stress one needs a model for the tissue response to strain. Linear elastic material is long known to be inadequate to describe soft tissue responses to mechanical loads. To describe the tissue behavior under some strain $\epsilon(t)$ we take the standard viscoelastic model after [9]:

$$
\frac{d}{d t} \sigma(t)+\frac{E \sigma(t)}{\mu}=\frac{E E_{0} \epsilon}{\mu}+\left(E+E_{0}\right) \frac{d}{d t} \epsilon(t)
$$

Solving symbolically for $\sigma(t)$ leads to an expression for stress relaxation:

$$
\sigma(t)=\epsilon_{0}\left(E_{0}+E e^{-E t / \mu}\right)
$$

with $\epsilon_{0}\left(E_{0}+E\right)$ the linear contribution to total stress and $\mu$ the relaxation time.

Under the assumption of tissue incompressibility and approximate spherical symmetry, the thickness of the thin tissue rim surrounding the myoma from the cavity side has to change with the increase of the pathology radius in order to compensate for the volume change. Perfectly elastic wall (hoop) stress in a thin-walled sphere is given as:

$$
\sigma(r)=1 / 2 \frac{p r}{-r+\sqrt{r^{2}+2 w_{0} r_{0}+w_{0}^{2}}}
$$

with $r_{0}$ and $w_{0}$ rest-state radius of the sphere and the thickness of its wall, respectively. Even if the strain-stress relation is linear, as seen in Fig. 11 (solid line), the wall stress is not, because of the variable wall thickness. Solution for a general (including viscous) stress will be of a similar form, multiplied by a 
relaxation factor (dashed line). What this means for our model is that we are able now to simulate a spectrum of tissue behaviors: from perfectly elastic $(\mu \rightarrow 0)$ to perfectly plastic $(\mu \rightarrow \infty)$, regulating the relaxation contribution with $\mu$. Of course, in general, these model parameters will be different for the tumor and the surrounding tissue. The resulting overall model depends on 5 unknowns: 3 concentrations (scalars) and 2 stresses (tensors). The model parameters that we have used in the actual implementation are discussed below.

\subsection{Estimation of the Model Parameters}

Based on measurements of the average thickness $d_{v}$ of the viable rim of in vitro tumor spheroids in 1011, and estimates of oxygen consumption rates in [12]13] and diffusion coefficient in [1213, we can estimate the critical drop in the oxygen concentration which leads to hypoxia-induced growth factor production. By solving analytically a radially symmetric constant boundary reaction-diffusion problem in an idealized spherical geometry and using a linear oxygen reaction rate as before, we get an estimate for $c_{3}^{t h r}$ of $1.2 \times 10^{-3}\left(\mathrm{mlO}_{2}\right)(\mathrm{ml} \text { tissue })^{-1}$, which corresponds to approximately $31 \mathrm{mmHg}$. This seems reasonable, since the median partial pressure in breast cancers was $30 \mathrm{mmHg}$ as measured by [14. We were unable to find any measurements of the AGF uptake rate $R_{1}$ in the literature. We assume the value $R_{1}=10^{-6} s^{-1}$. Estimates for the diffusion coefficient for different AGFs are in the range of one to two orders of magnitude smaller than for oxygen, we use the value $D_{1}=10^{-10} \mathrm{~m}^{2} \mathrm{~s}^{-1}$ after [15]. In order to set the coupling factor $\alpha_{1}$, we measure the hypoxic area $H$ of the tumor in the initial configuration where we define $c_{1}=c_{0}=10^{-7}$ mole $/ m^{3}$ ([16]), i.e. $\alpha_{1}=H / c_{0}$ which is approximately $1 / 3$.

The parameters $D_{2}, k_{2}$ and $k_{0}$ are taken from [16. [17] measured the chemotactic coefficient $k_{0}$ and diffusion coefficient $D_{2}$ of migrating endothelial cells in gradients of aFGF. The measurements were however made with unconstrained endothelial cells. As pointed out by [16] these experimental conditions overestimate the random motility, therefore they chose a smaller value for $D_{2}$. The

chemotactic function $k\left(c_{1}\right)=\frac{k_{0} k_{1}}{k_{1}+c_{1}}$ is nearly constant for low AGF concentration $c_{1}$, but decreases for high concentrations in the range of $k_{1}$. The parameter $k_{1}$ is therefore chosen close to $c_{0}=10^{-7}$ mole $/ \mathrm{m}^{3}$ which is supposed to be a relatively high concentration, again measured for the chemoattractant aFGF. This receptor kinetic form reflects the more realistic assumption that chemotactic sensitivity decreases with higher AGF concentrations.

It has been shown in 10] that the oxygen consumption is reduced at lower oxygen concentrations. Therefore, we take a linear reaction rate in our model $R_{3}$. In order to estimate the oxygen delivered to the tissue by the vasculature in terms of the endothelial cell density, we make some simplifications. The oxygen flux through a segment of a vessel is approximately

$$
Q_{\mathrm{O}_{2}}=d S K_{\mathrm{O}_{2}}\left(P_{\mathrm{O}_{2}}^{\text {blood }}-P_{\mathrm{O}_{2}}^{\text {tissue }}\right) \frac{1}{w}
$$


where $P_{\mathrm{O}_{2}}^{\text {tissue }}$ is the oxygen partial pressure on the interface between the vessel and the tissue, $d S$ is the vessel surface, $w$ wall thickness, and $Q_{O_{2}}$ is the oxygen transported through the vessel wall ([13]). The partial pressure of the blood is given by $P_{\mathrm{O}_{2}}^{\text {blood }}$. The coefficient $K_{\mathrm{O}_{2}}$ is also known as Krogh's oxygen diffusion coefficient ([18]). Assuming the known mean radius $R$ of a capillary, and its thickness $w$, we can estimate oxygen source at the capillaries as

$$
S_{\mathrm{O}_{2}}=\frac{Q_{\mathrm{O}_{2}}}{V}=c_{2} \cdot \frac{2 R}{\left(R^{2}-(R-w)^{2}\right) w} K_{O_{2}}\left(P_{O_{2}}^{\text {blood }}-P_{O_{2}}^{\text {tissue }}\right)=c_{2} \cdot C_{0}
$$

In order to obtain an estimate for $\alpha_{3}$ in eq. 7 we multiply $C_{0}$ by the approximate diffusion length. For the mean tissue oxygen partial pressure we used the value measured in the breast by 14 to be $65 \mathrm{mmHg}$. The blood oxygen pressure was assumed to be that of arterial blood.

\section{Results}

A development of a virtual myoma is shown in Fig. 2. intensity-coded with stress, using identical scale to facilitate magnitude comparisons. The neoplasm started growing as a small oval pathology in myometrium and continues to grow into the surrounding tissue that is largely dissipating the generated strain energy. As shown, the tissue after being exposed to the critical stress of around $1 \mathrm{MPa}$ (Fig. 2e, value out of scale), gradually relaxes its stress allowing the pathology to continue expanding and therefore only weakly constraining its progression. The formation of an experimentally observed type I myoma is inevitable. Should the behavior of the surrounding tissue be dominated by elasticity (i.e., the expansion induced stress in the thin tissue slab would be mostly preserved), the stretched layer of the myometrium would very strongly constrain the growth. This is because the reaction force would be equal to - or greater than - the accumulated

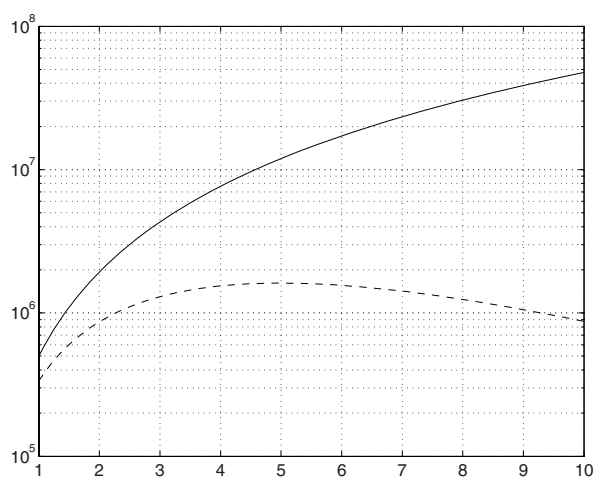

Fig. 1. Wall stress in the thin tissue layer accumulating the strain energy (solid line) and dissipating it (dashed line) 

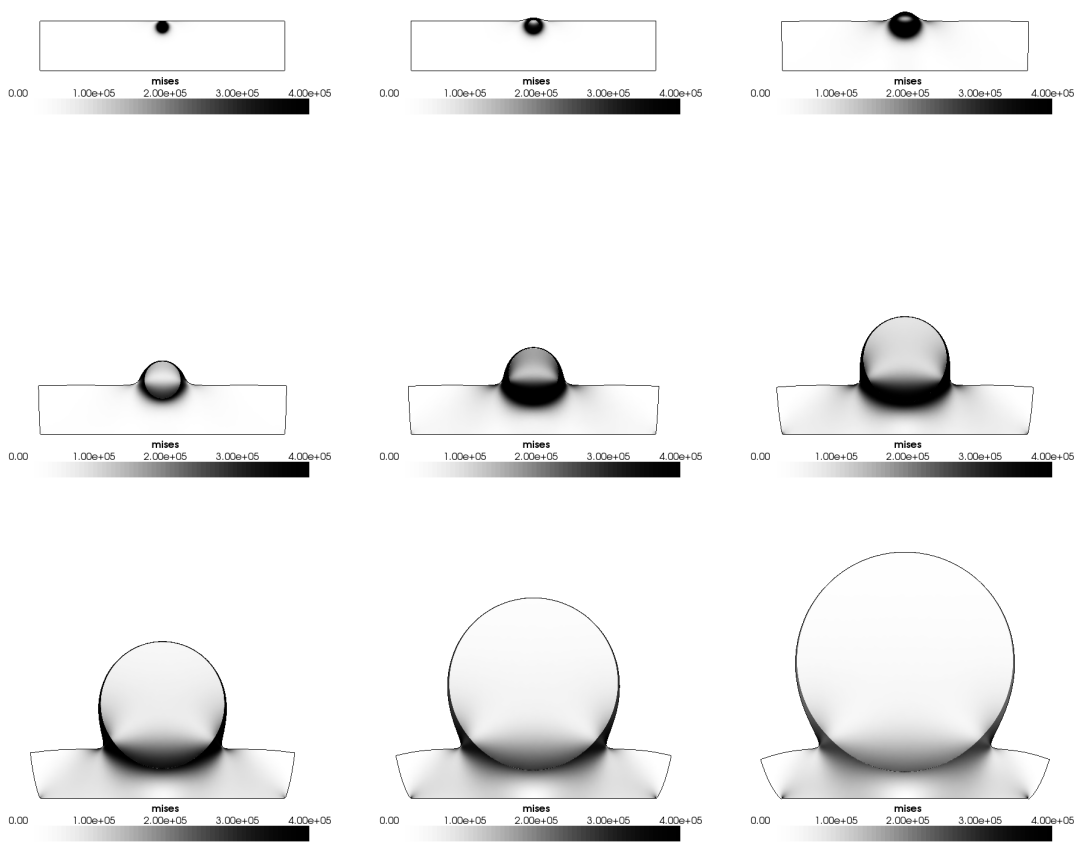

Fig. 2. Simulation results of a myoma (type I) developing at the border of the myometrium. Due to stress dissipation there is no significant resistance from the tissue layer on the cavity side (upwards on the image). As a result the pathology envelope forms an acute angle with the basement layer.

stress, comparable in magnitude to the elastic modulus of the pathology (c.f. Fig. (1). We have tested this scenario as well and found a myoma of type II with appearance identical to that in Fig. 20 only with much higher (accumulated) stress, constraining further growth. The myoma surfaces, surfaces only partially (if at all) or even protrudes back into the myometrium, depending on the initial starting position of the growth. All these scenarios are observed during myoma resection surgeries and can well be explained by our model (cf. [1]).

\section{Discussion and Outlook}

The findings resulting from our first computational experiments are in good agreement with observations on real cases. We are now in position to study e.g. how modifications of the vascular system by means of anti-angiogenic therapy could influence the dynamics and appearance of a myoma. We can also investigate how the different tissue properties determine if the myoma surfaces into the cavity or protrudes back into the myometrium. This and other in-silico experiments are under way and will be reported separately. 
A point of discussion is the viscoelasticity of the surrounding tissue. This could correspond to e.g. increased proliferation or loss of elasticity due to structural fiber damage under prolonged exposure to stress. From histological stains we know that the healthy myometrium mesh and the membranaceous endometrium may be stretched by a stiff growing myoma to the many fold of their original dimensions, coped by hypertrophy and/or hyperplasia. At a certain moment, thinning of healthy structures is not compatible with sufficient blood supply anymore, leading to necrosis in the top stressed area first, e.g. causing pain and bleeding disorders.

Obviously, our model cannot be free from simplifications. We currently only consider oxygen as the nutrient while tumor cells often do not require much oxygen for growth, but glucose, fat, and amino acids. Even when addressing only oxygen in our current model, we work under the assumption that the oxygen is delivered only to the tumor surface. We do not currently model the vascular penetration. Modeling the blood vessels explicitly as in 5] would allow making studies and predictions of the actual structure and function of the feeding vessels. Distinguishing between myometrium and endometrium is another extension that we consider in the next version.

The quantitative validation of the findings presented is currently prepared by comparisons to histologic stains and vascular corrosion castings of uteri containing myomas (c.f. 2]).

Acknowledgments. This work is part of the Swiss National Center of Competence in Research on Computer Aided and Image Guided Medical Interventions (NCCR Co-Me), supported by the Swiss National Science Foundation. We are indebt to prof. dr. med. Haymo Kurz from the Institute of Anatomy and Cell Biology, University of Freiburg, for numerous discussions and explanations.

\section{References}

1. Mencaglia, L., Hamou, J.E.: Manual of Gynecological Hysteroscopy. Endo-Press (2001)

2. Walocha, J.A., Litwin, J.A., Miodonski, A.J.: Vascular system of intramural leiomyomata revealed by corrosion casting and scanning electron microscopy. Hum. Reprod. 18(5), 1088-1093 (2003)

3. Szczerba, D., Szekely, G.: Simulating vascular systems in arbitrary anatomies. In: Duncan, J.S., Gerig, G. (eds.) MICCAI 2005. LNCS, vol. 3750, pp. 641-648. Springer, Heidelberg (2005)

4. Szczerba, D., Szekely, G., Kurz, H.: A multiphysics model of capillary growth and remodeling. In: Alexandrov, V.N., van Albada, G.D., Sloot, P.M.A., Dongarra, J. (eds.) ICCS 2006. LNCS, vol. 3992, pp. 86-93. Springer, Heidelberg (2006)

5. Lloyd, B., Szczerba, D., Székely, G.: A coupled finite element model of tumor growth and vascularization. In: Ayache, N., Ourselin, S., Maeder, A. (eds.) MICCAI 2007, Part II. LNCS, vol. 4792, pp. 874-881. Springer, Heidelberg (2007)

6. Gordon, V.D., Valentine, M.T., Gardel, M.L., Andor-Ardo, D., Dennison, S., Bogdanov, A.A., Weitz, D.A., Deisboeck, T.S.: Measuring the mechanical stress induced by an expanding multicellular tumor system: a case study. Experimental Cell Research 289(1), 58-66 (2003) 
7. Ausprunk, D.H., Folkman, J.: Migration and proliferation of endothelial cells in preformed and newly formed blood vessels during tumor angiogenesis. Microvascular Research (1977)

8. Cristini, V., Lowengrub, J., Nie, Q.: Nonlinear simulation of tumor growth. Journal of Mathematical Biology V46(3), 191-224 (2003)

9. Humphrey, J.D., DeLange, S.: An Introduction to Biomechanics. In: Solids and Fluids, Analysis and Design. Springer, Heidelberg (2004)

10. Freyer, J.P., Sutherland, R.M.: A reduction in the in situ rates of oxygen and glucose consumption of cells in EMT6/Ro spheroids during growth. J. Cell. Physiol. 124(3), 516-524 (1985)

11. Frieboes, H.B., Zheng, X., Sun, C.H., Tromberg, B., Gatenby, R., Cristini, V.: An integrated computational/experimental model of tumor invasion. Cancer Res. 66(3), 1597-1604 (2006)

12. Salathe, E.P., Xu, Y.H.: Non-linear phenomena in oxygen transport to tissue. Journal of Mathematical Biology 30(2), 151-160 (1991)

13. Ji, J.W., Tsoukias, N.M., Goldman, D., Popel, A.S.: A computational model of oxygen transport in skeletal muscle for sprouting and splitting modes of angiogenesis. J. Theor. Biol. 241(1), 94-108 (2006)

14. Vaupel, P., Schlenger, K., Knoop, C., Höckel, M.: Oxygenation of human tumors: evaluation of tissue oxygen distribution in breast cancers by computerized o 2 tension measurements. Cancer Res. 51(12), 3316-3322 (1991)

15. Gabhann, F.M., Popel, A.S.: Interactions of VEGF isoforms with VEGFR-1, VEGFR-2, and neuropilin in vivo: a computational model of human skeletal muscle. Am. J. Physiol. Heart Circ. Physiol. 292(1), H459-H474 (2007)

16. Anderson, A.R.A., Chaplain, M.A.J.: Continuous and discrete mathematical models of tumor-induced angiogenesis. Bulletin of Mathematical Biology V60(5), 857899 (1998)

17. Stokes, C.L., Rupnick, M.A., Williams, S.K., Lauffenburger, D.A.: Chemotaxis of human microvessel endothelial cells in response to acidic fibroblast growth factor. Lab. Invest. 63(5), 657-668 (1990)

18. Ursino, M., Giammarco, P.D., Belardinelli, E.: A mathematical model of cerebral blood flow chemical regulation-Part I: Diffusion processes. IEEE Trans. Biomed. Eng. 36(2), 183-191 (1989) 